OPEN ACCESS

Edited by:

Aiping Lu,

Hong Kong Baptist University,

Hong Kong

Reviewed by:

Wei Song,

Peking Union Medical College Hospital (CAMS), China

Haolong Liu,

Peking University Health Science

Center, China

*Correspondence:

Xiaomei Guo

xiaomguo@yeah.net

Specialty section:

This article was submitted to

Ethnopharmacology,

a section of the journal

Frontiers in Pharmacology

Received: 01 August 2018 Accepted: 24 September 2018

Published: 14 November 2018

Citation:

Lu L, Sun X, Chen C, Qin Y and Guo X (2018) Shexiang Baoxin Pill, Derived From the Traditional Chinese Medicine, Provides Protective Roles

Against Cardiovascular Diseases.

Front. Pharmacol. 9:1161

doi: 10.3389/fphar.2018.01161

\section{Shexiang Baoxin Pill, Derived From the Traditional Chinese Medicine, Provides Protective Roles Against Cardiovascular Diseases}

\author{
Li Lu', Xiaodong Sun ${ }^{2}$, Chen Chen ${ }^{1}$, Yating Qin ${ }^{1}$ and Xiaomei Guo ${ }^{1 *}$ \\ 1 Department of Cardiology, Tongji Hospital, Tongji Medical College, Huazhong University of Science and Technology, \\ Wuhan, China, ${ }^{2}$ Department of Orthopedics, Puai Hospital, Tongji Medical College, Huazhong University of Science \\ and Technology, Wuhan, China
}

Shexiang Baoxin Pill (SBP), derived from the traditional Chinese medicine, has been broadly applied for the treatment of cardiovascular diseases including coronary heart disease, heart failure, and hypertension in East Asia for decades. Emerging pharmacological studies have revealed that SBP displays pleiotropic roles in protecting the cardiovascular system, as seen by the promotion of angiogenesis, amelioration of inflammation, improvement of endothelium dysfunction, mitigation of dyslipidemia, repression of vascular smooth muscle cell proliferation, and migration and restraint of cardiac remodeling. In terms of clinical practice, the clinical trials and meta-analyses have proved the efficacy and safety of SBP. In this review, we, for the first time, systematically summarize the cardioprotective effects and underlying mechanisms of SBP and provide novel insights into future research directions of SBP based on the experimental and clinical perspectives. Keywords: Shexiang Baoxin Pill, traditional Chinese medicine, cardiovascular disease, angiogenesis, cardiac
remodeling

\section{INTRODUCTION}

It is well-recognized that cardiovascular diseases (CVDs) are regarded as the leading cause of death worldwide. Despite the rapid development of western medicines in treating CVDs, the mortality of CVDs still remains high. In developed countries of North America, nearly 170 health losses per 100000 were attributed to CVDs in 2015 (Roth et al., 2017). Moreover, in developing countries of Asia, the mortality of CVDs was 298 cases per 100000 in the same year (Ma et al., 2018). It is imperative to find other remedies for improving the treatment and prognosis of CVDs. Complementary and alternative therapeutics such as traditional Chinese medicines (TCMs) are emerging as crucial protective agents for the cardiovascular system, considering their effective roles in inhibiting pathogenic events of CVDs (Liu and Huang, 2016; Hao et al., 2017).

Shexiang Baoxin Pill (SBP), a classic patent medicine derived from the TCM formula Suhexiang Pill of Song Dynasty in China, has been extensively used for the prevention and treatment of CVDs, such as unstable angina, myocardial infarction (MI), and heart failure (HF) (Zhou et al., 2016; Dong T. et al., 2018). There are seven TCM materials contained in the SBP: Artificial Moschus, Radix Ginseng (Root of Panax ginseng C.A.Mey.), Calculus Bovis Artifactus, Cortex Cinnamomi (Bark of Cinnamomum cassia Presl), Styrax (balsam of Liquidambar orientalis Mill.), Venenum Bufonis, 
and Borneolum Syntheticum (Resin of Dryobalanops aromatica C.F.Gaertn) (Figure 1). From the perspective of preclinical studies, SBP has demonstrated therapeutic effects on CVDs via various beneficial mechanisms including regulating angiogenesis and coronary artery dilation, repressing inflammation and oxidation stress, improving lipid metabolism, and protecting vascular endothelium (Ning et al., 2011; Xu et al., 2011; Zhang et al., 2017; Wei et al., 2018). Additionally, in terms of clinical practice, the cardiovascular protective roles of SBP have been proven by several randomized controlled trials and expert consensuses on SBP treatment of CVDs (Zhou et al., 2016; Dong T. et al., 2018; Fan et al., 2018). In the following sections, we provide an overview of basic experiments and clinical studies on the cardiovascular effects of SBP and the underlying mechanism profiles.

\section{PHARMACOLOGICAL FEATURES OF SBP}

\section{Active Components}

Using chromatography and mass spectrometry techniques, more than 70 non-volatile and 40 volatile compounds in SBP have been identified by in vitro studies, including cholic acid, deoxycholic acid, cinobufagin, and ginsenoside Rb1 (Liu et al., 2015; Lv et al., 2017). The internal related substances after oral administration of SBP have also been widely analyzed during the past years. Jiang et al. (2009) found that 17 compounds and 4 metabolites were present in the plasma of rats after SBP uptake, which contained gamabufotalin, resibufaginol, ginsenoside $\mathrm{Re}$, chenodeoxycholic acid, and 17hydroxyprogesterone. Using a sensitive mass spectrometry method, another study determined 4 volatile components in rat plasma after gastric perfusion with SBP, including isoborneol, borneol, muscone, and cinnamaldehyde (Chang et al., 2014). Moreover, as the main constituents with therapeutic roles and cardiotoxic effects in SBP separately, ginsenosides from Radix Ginseng and bufadienolides from Venenum Bufonis were rapidly absorbed into the blood, and the pharmacokinetic characteristics of their metabolites have been investigated for improving the efficacy and safety of drug application (Table 1; Huang et al., 2015; Peng et al., 2015; Tao et al., 2017).

\section{Herb-Drug Interaction}

Shexiang Baoxin Pill has been prescribed alone or in combination with other drugs for the treatment of CVDs over the years (Chen et al., 2018; Fan et al., 2018). Considering that SBP affects the activities and levels of several enzymes which regulate the metabolisms of some clinical drugs, combination therapies combining SBP with modern drugs have gained increasing attention for reducing the potential of adverse effects (Shen et al., 2016). For instance, cytochrome P450s (CYP450s) represent a group of hemeproteins catalyzing biotransformation of the majority of medications and SBP has shown to modulate the activities and expressions of catalytic enzymes among CYP450s, thereby suggesting that SBP might affect pharmacokinetic profiles of drugs metabolized by CYP450s (Jiang et al., 2012; Shen et al., 2016). According to a study reported by Tao et al., increased $\mathrm{C}_{\max }$ and $\mathrm{AUC}_{(0-\mathrm{t})}$ and reduced $\mathrm{T}_{1 / 2}$ and $\mathrm{T}_{\max }$ of simvastatin were seen in plasma after simvastatin was co-administrated with SBP (Tao et al., 2016). Thus, changes in drug metabolism parameters induced by the combination therapies and related mechanisms should be further explored to guide clinical drug applications.

\section{PROTECTIVE MECHANISMS OF SBP}

\section{Promotion of Angiogenesis}

Angiogenesis is a pivotal protective action in the ischemia pathologic environment, which induces the formation of new capillaries and ensures blood and oxygen supply that accelerates damage restoration (Sun et al., 2018). Previous studies have established that SBP is capable of alleviating arterial obstructive disorders via facilitating angiogenesis activities (Zhang et al., 2017; Guo et al., 2018). Huang F. et al. (2017) found that SBP significantly reduced the infarction area and enhanced cardiac functions of Sprague-Dawley rats with MI. They demonstrated that this cardioprotective role was attributed to SBP-induced angiogenesis in pert-infarct areas, and the underlying mechanism was associated with SBP-mediated level increase of serum 20-hydroxyeicosatetraenoic acid, which upregulated vascular endothelial growth factor (VEGF) expression and then boosted circulating endothelial progenitor cells (EPCs) to proliferate and accumulate to the injured region for participating in angiogenic events (Huang F. et al., 2017). In addition, under the stimulation of sheer stress, SBP enabled the EPCs to possess increased abilities of angiogenesis by means of inducing endothelial nitric oxide synthase (eNOS) expression and subsequent generation of nitric oxide (NO) that was considered as an agonist of vessel formation (Li G. et al., 2015). Moreover, cinnamaldehyde, a bioactive ingredient from SBP, has been proven to exert pro-angiogenic properties by heightening the proliferation, mobilization, and tube formation of endothelial cells (ECs) through stimulating specific molecules and pathways responsible for pro-angiogenic processes such as VEGF, PI3K/Akt, and MAPK signaling cascade (Yuan et al., 2018). Other research indicated that SBP and its component ginsenosides shared similar pharmacological effects as cinnamaldehyde on ECs, whereas the possible mechanisms of the results remained unknown, probably linked to angiogenic modulators including LATS1/2/YAP/CTGF and VEGFR1 cascade which were targeted by SBP (Lv et al., 2014; Fang et al., 2017; Wu et al., 2018). In an animal experiment regarding MI, SBP decreased the MI area and increase microvessel count via improving angiogenesis, as evidenced by elevation of VEGF and basic fibroblastic growth factor (Wang et al., 2002). On an interesting note, some scholars declare that SBP repress angiogenic processes in the atherosclerotic lesions for enhancing the stabilization of plaques (Li et al., 2006; Shen et al., 2010). This phenomenon indicates that the effects produced by SBP on angiogenesis might vary among different pathophysiological environments, and illuminating the precise mechanisms is valuable for 


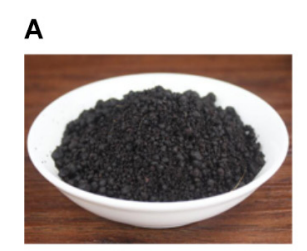

Artificial Mouchus

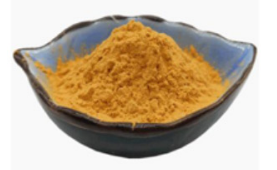

\section{Calculus Bovis Artifactus}

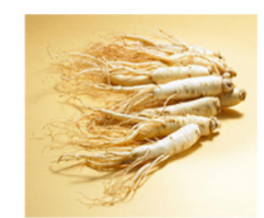

\section{Radix Ginseng}

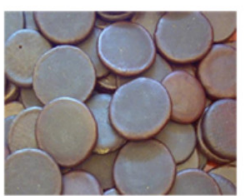

B

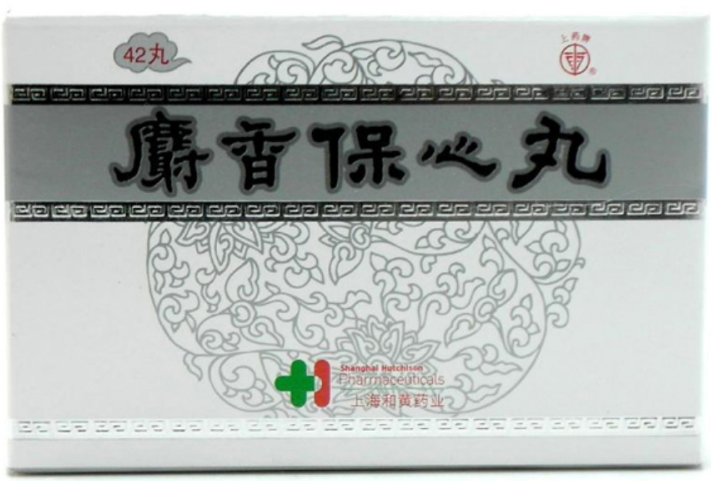

Borneolum Syntheticum

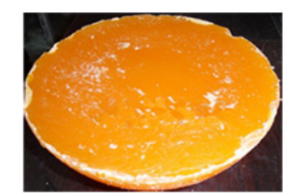

Styrax

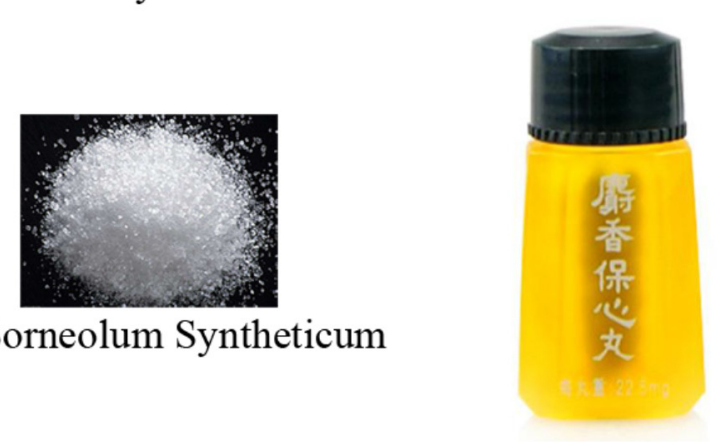

\section{Cortex Cinnamomi}

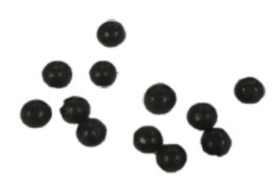

\section{Venenum Bufonis}

FIGURE 1 | The components included in Shexiang Baoxin Pills and the related Chinese patent medicine. (A) The morphology of seven raw medicinal materials which compose Shexiang Baoxin Pills. (B) The relevant patent medicine of Shexiang Baoxin Pills manufactured by Shanghai Hutchison Pharmaceuticals, which have been used in clinical practice for several years. The picture of this patent medicine has been permitted to be presented in the manuscript by Shanghai Hutchison Pharmaceuticals.

providing new insights into the protective roles of SBP against CVDs.

\section{Amelioration of Inflammation}

A substantial number of investigations have demonstrated that inflammation plays a vital role in the initiation and progression of CVDs. Inflammatory cells and their secreted cytokines destroy the integrity of vascular endothelium, which result in LDL$\mathrm{C}$ disposition and oxidation in the subendothelium and the transendothelial recruitment and foam cell transformation of macrophages, favoring the development of atherosclerosis (AS). In addition, the inflammatory state increases the consumption of energy and oxygen, which adds to the burden of the heart pumping blood, and leads to raised mortality of MI and chronic HF (Siti et al., 2015; Shapiro and Fazio, 2016). There is also evidence that inflammation is able to trigger cardiac arrhythmia by promoting electrical and structural remodeling (Hu et al., 2015). Then, inhibiting inflammation is a promising approach for treating CVDs Xu et al. (2011)
TABLE 1 | The main chemical components included in the raw medicinal materials of SBP.

\begin{tabular}{|c|c|c|}
\hline Medicinal materials & Bioactive components & Reference \\
\hline Artificial Moschus & Muscone, testosterone & Fang et al., 2017 \\
\hline Radix Ginseng & $\begin{array}{l}\text { Ginsenoside Ra1/2, Rb1/2/3, } \\
\text { Rc, Rd, Re, Rf, and Rg1/2/3 }\end{array}$ & Tao et al., 2017 \\
\hline $\begin{array}{l}\text { Calculus Bovis } \\
\text { Artifactus }\end{array}$ & $\begin{array}{l}\text { Cholic acid, deoxycholic acid, } \\
\text { ursodeoxycholic acid, } \\
\text { chenodeoxycholic acid, } \\
\text { hyodeoxycholic acid, bilirubin, } \\
\text { and cholesterol }\end{array}$ & Jiang et al., 2009 \\
\hline Cortex Cinnamomi & $\begin{array}{l}\text { Cinnamaldehyde and cinnamic } \\
\text { acid }\end{array}$ & Lv et al., 2017 \\
\hline Styrax & Benzyl benzoate & Liu et al., 2015 \\
\hline Venenum Bufonis & $\begin{array}{l}\text { Cinobufagin, resibufogenin, } \\
\text { resibufagin, gamabufotalin, } \\
\text { bufalin, } 1 \beta \text {-hydroxylbufalin, } \\
\text { arenobufagin, bufotalin, } \\
\text { telocinobufagin, and telibufagin }\end{array}$ & Tao et al., 2017 \\
\hline Borneolum & Borneol and isoborneol & Chang et al., 2014 \\
\hline
\end{tabular}

Syntheticum 
elaborated that pretreatment with SBP, in a dose-dependent manner, reversed the increment of MCP-1 and IL- 6 in ECs induced by $\mathrm{H}_{2} \mathrm{O}_{2}$ by lowering the content of inflammatory transcriptional factor NF-кB. In terms of anti-AS, Tao et al. observed that SBP alleviated the atherogenesis in rabbits with a high fat diet, accompanied by decrement of TNF$\alpha$, IL-6, and IL-8, which was partially related with its antiinflammatory activity by blocking $\mathrm{Ca}^{2+} / \mathrm{CaMKII/TLR/NF- \kappa B}$ axis (Tao et al., 2015). With network pharmacology-based methods, several inflammation related molecules were identified to be targeted by SBP, including ICAM-1, COX-2, TLR4, and VCAM-1 (Fang et al., 2017). Mounting evidence in literature increasing evidence in the published papers have suggested that SBP is capable of mitigating the progression of hypertensive nephropathy and cardiotoxicity by diminishing the tissue inflammatory responses, as seen by the decline in TNF- $\alpha$, iNOS, TGF- $\beta 1$, IL-1 $\beta$, and ICAM-1 (Hu et al., 2009; Tian et al., 2011). In addition, damage of cardiac cells triggered by high glucose was ameliorated when cells co-incubated with SBP, which was due to the suppression of p38 MAPK and NF-кB pathway, thereby indicating the potentials of SBP in diabetic cardiomyopathy treatment (Yang et al., 2016). Studies analyzing serum metabolomics reported that the efficacy of SBP in MI therapy was partly ascribed to the anti-inflammatory abilities of SBP, given that the detected biomarkers involved in inflammation such as PGE2 and 12(S)-HETE were down-regulated by SBP (Xiang et al., 2012, 2013). Moreover, SBP was found to depress the incidence of arrhythmias and improved ventricular remodeling in rats with MI by decreasing the expression of inflammation cytokines including IL-18 and TNF- $\alpha$ in the damaged areas (Xu et al., 2015; Yang B. et al., 2015). Metabolic syndrome (MS), characterized by dyslipidemia, hypertension, and hyperglycemia, is thought to be a key contributor to CVDs. It was revealed that SBP provided the cardioprotective actions by abrogating the development of MS, impeding NF- $\kappa$, TNF- $\alpha$, and IL6 expression and raising the level of IL-10 and PPAR- $\gamma$, an anti-inflammatory factor encumbering the activation of NF- $\kappa \mathrm{B}$ (Li Y. et al., 2015; Wei et al., 2018). Therefore, SBP exerts anti-inflammatory roles, mainly by decreasing the expression and activation of $\mathrm{NF}-\kappa \mathrm{B}$, thereby suggesting the potential contribution of NF- $\kappa \mathrm{B}$ suppression to the cardioprotective effects of SBP.

\section{Improvement of Endothelium Dysfunction}

Recovering and maintaining the normal function of endothelium has been proposed as a promising approach for treating CVDs, since dysfunction of endothelium is a pivotal pathogenic event of disease development of the cardiovascular system. When exposed to diverse stimuli, the endothelium barrier is damaged and its permeability is increased, which accelerates the accumulation of lipids and leukocytes into the tunica intima, thereby facilitating vascular stenosis followed by the occurrence of ischemic heart disease and stroke (Gimbrone and Garcia-Cardena, 2016). As endothelium dysfunction leads to the reduction of some endothelial-dependent vasoactive substances such as NO, the vasodilation property is impaired and related vascular stiffness is raised, thereby elevating the blood pressure and aggravating hypertension (Oparil et al., 2018). Reactive oxygen species (ROS) and oxidative stress are known as the principal pathological elements implicated in endothelial injury (Ungvari et al., 2018). Emerging evidences have clarified that SBP could diminish $\mathrm{H}_{2} \mathrm{O}_{2}$-provoked ECs apoptosis and improve cell proliferative activity. The underlying mechanism involves anti-oxidative effects of SBP-induced level increase of SOD and expression reduction of MDA and NADPH oxidase p22phox subunit (Ning et al., 2011). Additionally, SBP was reported to enhance the production of NO via boosting eNOS activity, which might be another mechanism by which SBP protected ECs from ROS-induced damage, considering the fact that NO played key roles in regulating anti-apoptotic and anti-oxidative abilities of ECs (Li et al., 1999; Ungvari et al., 2018). In an experiment model of cerebral ischemia reperfusion injury, SBP was found to elevate the Bcl-2 level and lower the caspase-3 level for protecting the brain cells, but the relevant apoptotic pathways modulated by SBP involved in improvement of endothelium dysfunction are still required to be elucidated (Chen et al., 2008). Furthermore, SBP has been revealed to exhibit the therapeutic endothelial protection in patients with CVDs by raising NO and SOD and reducing ET-1 and MDA (Zhang Y. et al., 2016).

\section{Mitigation of Dyslipidemia}

Both the basic research and clinical trials have documented that disorder of lipid metabolism is deeply associated with progression of AS and its sequelae (Shapiro and Fazio, 2016). Agents lowering lipids in the circulation display great implications in preventing and treating atherogenesis (Hurtubise et al., 2016). $\mathrm{Yu}$ et al. discovered that SBP lessened the contents of blood TG, TC, and LDL-C, while augmenting the level of HDL$\mathrm{C}$ in rabbits that received high fat diets accompanied by the decline in thickness of plaques and intima-media thickness in the abdominal aorta, which may possibly be linked to SBPevoked decreased lipid sedimentation in the vascular wall ( $\mathrm{Yu}$ et al., 2012a). Furthermore, the mechanism underlying SBP modulating dyslipidemia has also been investigated. There was evidence showing that SBP was able to elevate the expression of key molecules required for the pathways of fatty acid oxidation, including AMPK, PPAR- $\alpha$, and PGC- $1 \alpha$, resulting in catabolism and decline of TG (Li Y. et al., 2015; Wei et al., 2018). As PPAR- $\gamma$ signaling is responsible for HDL-C biosynthesis in the liver and release for circulation, SBP has been shown to promote PPAR- $\gamma$ expression in cardiac tissues, and the influence of SBP on PPAR- $\gamma$ level in hepatocytes should be analyzed ( $\mathrm{Li}$ $Y$. et al., 2015). In addition, histological examinations revealed that SBP significantly mitigated AS development and weakened the expression of LOX-1 in atheroma lesions (Zhu et al., 2008). As LOX-1 belongs to the group of scavenger receptors which promote macrophages to uptake ox-LDL and switch into foam cells, blockade of foam cell formation might be another important mechanism of SBP for delaying AS progression. As LOX-1 belongs to the group of scavenger receptors promoting macrophages uptake of ox-LDL and switch into foam cells, 
these findings suggest that a blockade of foam cell formation is another important mechanism of SBP for delaying AS progression.

\section{Proliferation and Migration Repression of Vascular Smooth Muscle Cells}

Uncontrolled proliferation and migration of vascular smooth muscle cells (VSMCs) is a hallmark of CVDs such as AS, restenosis after percutaneous coronary intervention (PCI), and hypertension. Under normal situations, VSMCs keep a resting status termed as contractile phenotype in the tunica media, but they change into the unchecked synthetic phenotype and then proliferate and mobilize into the subendothelial layer, thereby leading to intimal thickening, in response to diverse stimuli such as inflammatory cytokines and stress damage. After switching into the synthetic state, VSMCs possess lessened levels of differentiation biomarkers including $\alpha$-SMA, SM22 $\alpha$, and SM-MHC, except for exhibiting enhanced growth and migration capabilities (Bennett et al., 2016; Durgin and Straub, 2018; Liu et al., 2018). Che et al. (2010) reported that the expression of $\alpha$-SMA and SM-MHC in VSMCs was elevated after co-administration of VSMCs with different concentrations of SBP. Similarly, Zhang et al. (2014) showed that SBP increased the positive rate of VSMCs expressing $\alpha$-SMA and SM-MHC while arresting the cell cycle at G1 phase. These findings hint that SBP might perform anti-proliferative functions on VMSCs by expediting the phenotypic switch from the synthetic to contractile state. With respect to the effects of SBP on VSMCs mobilization, another study revealed that SBP could prevent restenosis following stenting by impeding neointimal formation by repressing the movement of VSMCs (Yu et al., 2012b).

\section{Restraint of Cardiac Remodeling}

In view of the limited regenerative capacity of mature cardiomyocytes, loss of functional myocardial cells induces the remaining myocardium to endeavor and compensate for the decrease of contractile function and generate excessive extracellular matrix, leading to myocardial hypertrophy, structure disorder and fibrosis, termed as cardiac remodeling, a common pathological change in HF post-MI, hypertensive and diabetic cardiomyopathy (Dong Y. et al., 2018). A study monitoring the roles of SBP in the cardiac pathological changes of diabetic rats showed that SBP significantly alleviated myocardial interstitial fibrosis, reversed disorder of fiber arrangement and mitochondrial swelling, and improved the systolic and diastolic functions, which was probably due to the depressed levels of TGF- $\beta 1$ and Ang II in local tissues (Liu et al., 2012). $\mathrm{Xu}$ and Zhang (2016) also discovered that SBP could prohibit the activation of TGF- $\beta 1 /$ Smads pathway to encumber the development of hypertension-provoked myocardium fibrosis. In addition, according to Jiang et al. (2011), a few downregulated biomarkers comprising of corticosterone, aldosterone, and cortisol were detected in the MI rats' serum treated with SBP. Since these affected substances were metabolites of steroid hormone biosynthesis and facilitated the occurrence of hypertrophy, interruption of the pathway of steroid hormone generation appeared to be responsible for the suppressive effects of SBP on cardiac hypertrophy (Jiang et al., 2011). Moreover, previous evidence suggests that restoring the balance between MMP-9/TIMP-1 was a critical mechanism underlying SBP-impeded cardiac interstitium remodeling in rabbits with MI-evoked HF (Huang et al., 2011). Considering that aberrant activation of nerval and humoral factors had been indicated to perform positive effects on HF progression, gavaging with SBP increased the left ventricular ejection fraction (LVEF) and improved cardiac functions of rats suffering from HF. The mechanism was likely to be attributed to signal transduction modulation of the sympathetic and renin-angiotensin system, as seen by SBP-induced level alterations of $\alpha_{1}$ an $\beta$ adrenergic receptors and angiotensin II (Ang II) type 1 and type 2 receptors (Cao et al., 2011; Li et al., 2012).

Furthermore, pharmacological experiments have depicted that SBP could attenuate the dysfunction of energy metabolism aroused in MI animal models while reducing the size of necrotic and fibrotic tissues (Fan et al., 2011; Xiang et al., 2012, 2013). Because of the vital roles of energy metabolism in keeping the organs functioning adequately, SBP has the potential to prevent the aggravation of cardiac remodeling by mediating the energy metabolism pathways.

\section{SBP TREATMENT FOR CVDS}

\section{Unstable Angina}

It is well-known that acute coronary syndrome (ACS) can be divided into two categories: unstable angina and $\mathrm{MI}$. Clinical observations and retrospective analyses have clarified the effectiveness of SBP in treating unstable angina pectoris. Xing et al. reported that SBP had more advantages in depressing the frequency of an angina attack than isosorbide mononitrate tablets in patients with unstable angina (Xing et al., 2015). Yang and Zhang (2016) observed the safety and efficacy of SBP in elderly patients. They showed that SBP reversed the abnormal manifestation of electrocardiogram (ECG), improved the hemodynamics and coagulation risk, and enhanced the vasodilatation ability without producing obvious adverse effects, thereby likely lowering the incidence of acute thrombotic events. Several scholars implemented the trials involving combination drug therapy and found that the therapeutic profiles of the combination strategies amalgamating SBP with conventional medications were superior to that of the routine western drugs alone, which was explained by decrease of frequency and duration of angina occurrence, decrement of nitroglycerin consumption, restoration of ECG presentation, and elimination of serum inflammatory and oxidative factors (Zhang, 2013; Xie and Wang, 2016; Jiang et al., 2017).

\section{Myocardial Infarction}

In the cases of MI, a lot of evidence has demonstrated that SBP exhibits high efficiency in disease therapy. For example, studies compared SBP combined with conventional remedies versus conventional therapies alone for management of STsegment elevation MI after PCI. These studies showed that SBP 
remarkably improved LVEF and NT-proBNP level, diminished the contents of myocardial injury markers including $\mathrm{CK}$, cTNT, and cTNI, and decreased the overall rate of adverse cardiovascular events (Liu, 2016; Zhang and Guan, 2016). In a meta-analysis evaluating the effects of SBP in treating non-STsegment elevation MI, decline in risks of cardiovascular events and hospitalizations and remission of angina symptoms and abnormal ECG findings with good tolerance were observed in patients after long-term oral administration of SBP (Zhou et al., 2016). Moreover, according to Li et al. (2018), SBP proved to enhance the blood flow of stented artery and related myocardial perfusion following stent implantation, thereby hinting the remedial hope of SBP in slow-flow/no-flow post-PCI, which was possibly linked to protective roles of SBP against endothelium dysfunction. It was demonstrated that severe inflammatory reactions occured during the $\mathrm{MI}$ episode, which could induce the lethal arrhythmia (Hu et al., 2015; Siti et al., 2015). There was evidence that SBP improved QT interval dispersion and the heart rate variability, decelerating the incidence of ventricular arrhythmia in patients experiencing MI (Luo, 2015; Feng et al., 2017). Together with the fact that SBP serves as an antiinflammatory agent, SBP appears to encumber the occurrence of MI-related arrhythmia via abrogating inflammatory responses, while the specific mechanisms warrant further exploration. Additionally, Zhang L. et al. (2016) found that SBP was able to mitigate clopidogrel resistance in ACS participants by improving the platelet aggregation rate. With regard to thrombolytic therapy, Chen and Ma (2013) and Yang F. et al. (2015) drew a conclusion that SBP, when added to the routine thrombolytic program, produced better therapeutic influences on MI patients, as indicated by decrement of blood LDL and TG, enhancement of cardiac function, attenuation of myocardial impairment, and protection of vascular endothelium.

\section{Heart Failure}

As the advanced stage of multiple CVDs, HF with high death rates pose a great threat to quality of life, it also places a heavy burden on the whole of society (Roth et al., 2017). SBP has been broadly applied as an adjuvant treatment in HF and the corresponding pharmacological effects have been extensively investigated in recent years. Ding et al. assessed the clinical efficacy of SBP on the treatment of chronic ischemic HF. Following up after 6 months, the authors discovered that SBP substantially elevated the level of LVEF and 6-min walk distance (6-MWD) and depressed the value of BNP (Ding et al., 2013). In the cases of coronary heart disease (CHD)-related HF, a combination of SBP and conventional drugs exerted preferable effects compared to conventional medications, shown by the normalization of indicators reflecting cardiac function including LVEF, left ventricular end diastolic and systolic dimension, and cardiac output (CO), and decrease of specific HF biomarker NT-proBNP (Li R. et al., 2015; Huang, 2017). Additionally, the pooled data of a meta-analysis completed by Dong T. et al. (2018) mentioned that SBP was demonstrated to be effective and safe in suppressing HF progression, on account of the increment of LVEF, CO, stroke volume, and 6-MWD and decrement of BNP and NT pro-BNP without apparent side effects. In regard to HF with normal ejection fraction, SBP along with conventional treatment showed a more favorable impact than the conventional strategy on enhancing exercise tolerance, cardiopulmonary function, and life quality of patients (Huang M. et al., 2017). Kong et al. (2015) observed the therapeutic effectiveness of SBP on acute HF post-MI and reported that BNP levels and rates of all-cause death and cardiac death were lowered and the LVEF value was lifted after 2 months of SBP administration. Moreover, emerging evidence has established that some chemotherapy drugs exhibit cardiotoxicity and could impair the cardiac function, causing patients to suffer from HF (Moslehi et al., 2017). Recently, it was shown that SBP alleviated damages of myocardium and cardiac function triggered by doxorubicin via unknown mechanisms, as seen by improved levels of cTNI, CK-MB, BNP, LVEF, and fractional shortening (Qi et al., 2017). Thus, given the above studies, SBP is worthy of clinical application for mitigating HF development and improving the prognosis of patients.

\section{Hypertension}

According to the latest epidemiological data, approximately one billion of the global population are diagnosed with arterial hypertension, with nearly an estimated 270 million affected cohort in China (Forouzanfar et al., 2017; Ma et al., 2018). SBP has been used as complementary agent for the treatment of hypertension and relevant complications in Chinese patients for decades. Huang (2009) indicated that SBP added to amlodipine produced clinical efficacy superior to amlodipine alone in depressing the systolic pressure and pulse pressure of patients with isolated systolic hypertension. Trials concerning the management of hypertension with left ventricular hypertrophy reported that adjunctive therapy of SBP greatly lowered blood pressure, relieved clinical symptoms such as palpitations, chest stuffiness, and anhelation, and improved left ventricular posterior wall thickness, left ventricular mass index, interventricular septal thickness, and ECG parameters (Chen et al., 2012; Zhang Y. et al., 2016). In addition, SBP along with routine medications displayed higher efficiency in anti-hypertensive and cardiotonic roles in cases of hypertension combined with HF (Liu et al., 2017).

\section{Safety and Adverse Effects}

Despite the beneficial roles of SBP in the treatment of CVDs, the clinical safety and potential adverse reactions of SBP cannot be ignored. Previous toxicologic studies showed that TG levels and coagulation parameters were changed within a short time in very few female rats after oral administration with SBP at nearly 90 multiples of the human dosage, which implies that it might be advisable to monitor coagulation indexes and blood lipids of female patients. The side effects of SBP mainly include tongue numbness, nausea, emesis, rashes, dizziness, and palpitations. Several clinical trials have confirmed that the incidence rate of adverse effects is less than $0.01 \%$ and patients tolerate these side effects well. In addition, considering the potential cardiotoxicity of Venenum Bufonis in SBP, there is evidence indicating that other medicinal materials in SBP markedly alleviate the toxicity of Venenum Bufonis, thereby facilitating the safety of SBP in clinical practice (Wei et al., 2015). 
TABLE 2 | The therapeutic effects of SBP on the management of CVDs.

\begin{tabular}{|c|c|c|}
\hline Clinical application & Therapeutic effects & Reference \\
\hline \multirow[t]{4}{*}{ Unstable angina } & Angina symptom, nitroglycerin consumption $\downarrow$ & Xing et al., 2015 \\
\hline & Abnormal ECG parameters, hemodynamics, coagulation risks $\downarrow$ & Yang et al., 2016 \\
\hline & Hs-CRP, MMP-9, MDA, hcy, ET- $1 \downarrow$ & Xie and Wang, 2016; Zhang, 2013 \\
\hline & Vasodilatation, SOD and $\mathrm{NO} \uparrow$ & Zhang, 2013; Yang et al., 2016 \\
\hline \multirow[t]{6}{*}{ Myocardial infarction } & CK, CK-MB, LDH, BNP, NT-proBNP, cTNI, cTNT, H-FABP $\downarrow$ & Liu, 2016; Zhang and Guan, 2016 \\
\hline & MACE risks, hospitalization, angina symptom, ECG abnormity $\downarrow$ & Zhou et al., 2016 \\
\hline & QTd, QTcd, Platelet aggregation rate $\downarrow$ & Feng et al., 2017 \\
\hline & MMP-2, NLR, suPAR, IL-6, hs-CRP, LDL, TG, vWF $\downarrow$ & Chen and Ma, 2013; Li et al., 2018 \\
\hline & LVEF, TIMI flow, SOD, FMD $\uparrow$ & Yang F. et al., 2015; Li et al., 2018 \\
\hline & PNN50, RMSSD, SDNN, SDANN $\uparrow$ & Feng et al., 2017 \\
\hline \multirow[t]{4}{*}{ Heart failure } & BNP, ET-1, NT-proBNP, cTNI, CK-MB, H-FABP $\downarrow$ & Ding et al., 2013; Qi et al., 2017 \\
\hline & LVEDD, LVESD, FS, A/E $\downarrow$ & Li R. et al., 2015; Qi et al., 2017 \\
\hline & Dyspnea, all-cause death, cardiac death $\downarrow$ & Kong et al., 2015 \\
\hline & 6-MWD, LVEF, NO, CO, SV $\uparrow$ & Li R. et al., 2015; Huang, 2017 \\
\hline \multirow[t]{5}{*}{ Hypertension } & SBP, DBP, pulse pressure $\downarrow$ & Huang, 2009; Chen et al., 2012 \\
\hline & LVPWT, LVDD, IVST, LVMI $\downarrow$ & Zhang Y. et al., 2016 \\
\hline & FMD, EID, MDA, LPO, ET- $1 \downarrow$ & Zhang Y. et al., 2016 \\
\hline & Clinical manifestations $\downarrow$ & Chen et al., 2012 \\
\hline & $\mathrm{SOD}, \mathrm{TAC}, \mathrm{NO} \uparrow$ & Zhang Y. et al., 2016 \\
\hline
\end{tabular}

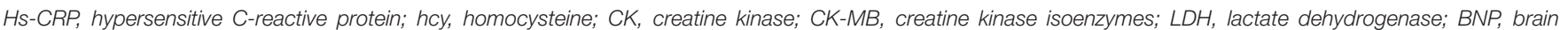

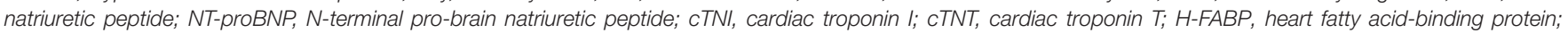

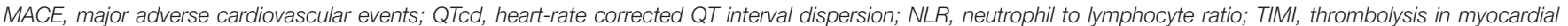

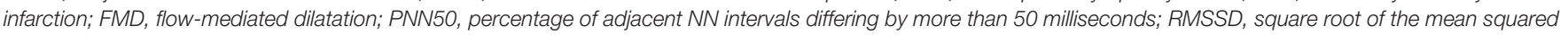

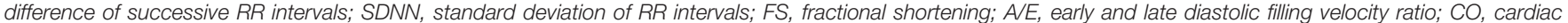

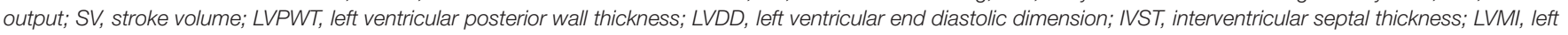
ventricular mass index; EID, endothelium-independent dilatation; TAC, total antioxidative capability.

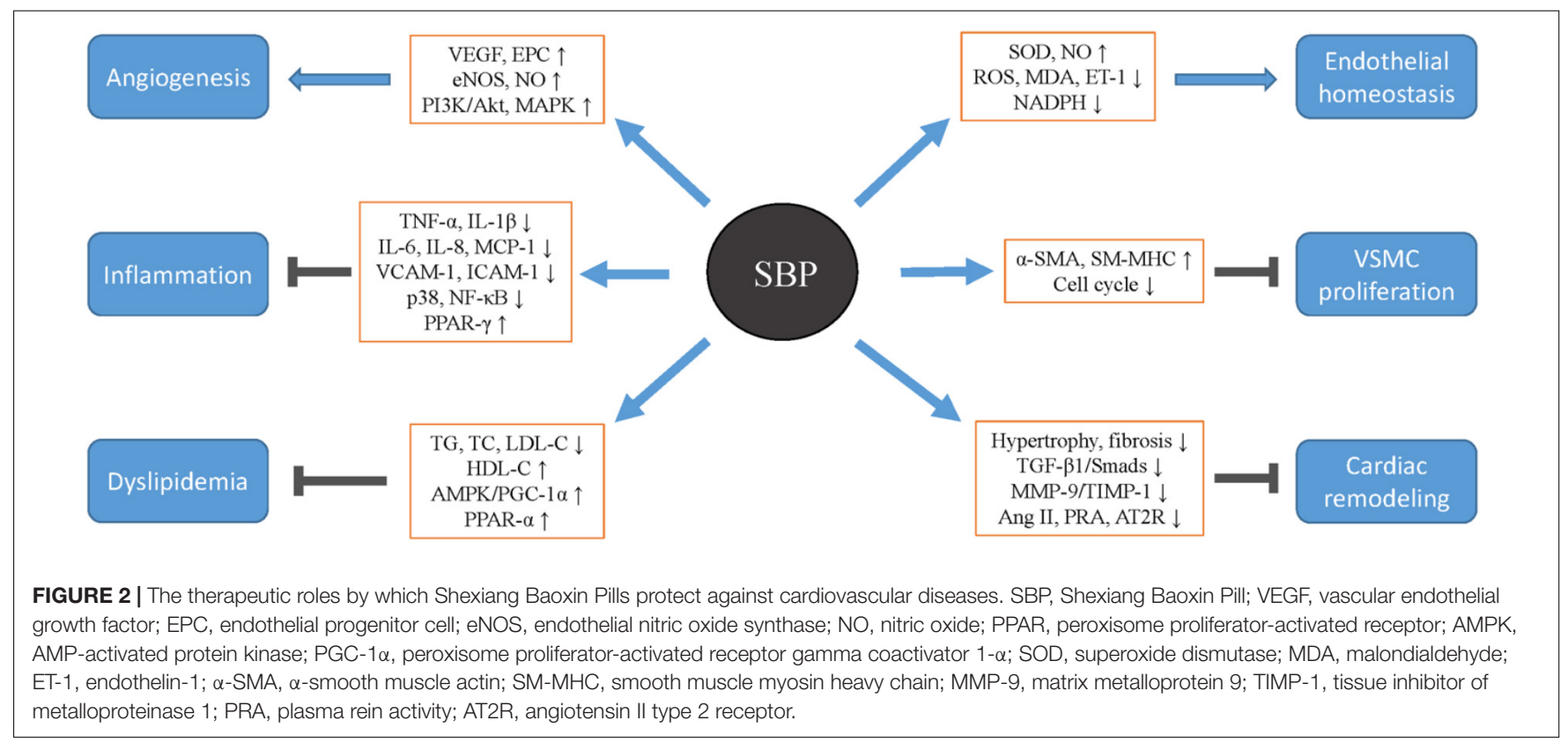

\section{CONCLUSION}

TCMs are widely associated with disease prevention and treatment (Liu and Huang, 2016). SBP, derived from the ancient TCM formula, has demonstrated to directly exert beneficial therapeutic effects on CVDs including CHD,
HF, and hypertension (Table 2), by means of promoting angiogenesis, inhibiting inflammation, improving endothelial function, and dyslipidemia, as well as interfering with VSMCs growth and cardiac remolding (Figure 2). In light of the pharmacological and therapeutic profiles in the cardiovascular system discussed above, SBP is deserving of further clinical 
promotion and application. At the same time, more experimental and clinical tests need to be conducted in the near future to provide more reliable evidence supporting SBP use. SBP is a mixture composed of seven raw medicinal materials and research currently mainly focuses on the pharmacokinetics of compounds in Radix Ginseng and Venenum Bufonis. The pharmacokinetic features of other ingredients such as the components in Artificial Moschus and Calculus Bovis Artifactus are scarce and should be analyzed to determine the effectiveness and safety of SBP. Moreover, to date, most basic studies investigating the protective mechanisms of SBP against CVDs detect the influences of SBP on the pathogenic phenomena and downstream effector molecules, while not exploring the key upstream signal pathways by which SBP affect disease development, leading to the ambiguity of functional signaling networks of SBP. It is therefore necessary for continual studies on the specific pathways involved in the cardioprotective mechanisms of SBP to lay a credible theoretical foundation. In addition, clinical trials observing the therapeutic efficacy of SBP on CVDs are characterized by small sample sizes,

\section{REFERENCES}

Bennett, M. R., Sinha, S., and Owens, G. K. (2016). Vascular smooth muscle cells in atherosclerosis. Circ. Res. 118, 692-702. doi: 10.1161/CIRCRESAHA.115. 306361

Cao, F., Li, Y., Liu, F., Bai, X., and Lv, Y. (2011). The effects of Shexiangbaoxin pills on the expression of cardiac angiotesin receptors in rat hearts with heart failure. J. Cardiovasc. Pulmonary Dis. 30, 241-245. doi: 10.3969/j.issn.1007-5062.2011. 03.017

Chang, W., Han, L., Huang, H., Wen, B., Peng, C., Lv, C., et al. (2014). Simultaneous determination of four volatile compounds in rat plasma after oral administration of Shexiang Baoxin Pill (SBP) by HS-SPDE-GCMS/MS and its application to pharmacokinetic studies. J. Chromatogr. B Analyt. Technol. Biomed. Life Sci. 963, 47-53. doi: 10.1016/j.jchromb.2014. 05.047

Che, X., Liang, S. X., Jiang, X., Gao, R., Zhang, Q., Qian, L., et al. (2010). Effects of She xiang bao xin wan on phenotype transformation of vascular smooth muscle cells. Chin. J. Pathophys. 26, 2149-2154. doi: 10.3969/j.issn.1000-4718.2010.11. 013

Chen, F., Liang, H., Tang, J., Huang, X., and Zhang, Y. (2012). Effects of Shexiang Baoxin Pill in adjuvant therapy for left ventricular hypertrophy in hypertension. Chin. J. Integr. Med. Cardiol. 10, 513-514.

Chen, K., Ge, J., and Zhang, M. (2018). Guideline of diagnosing and treating acute myocardial infarction by integrative medicine (Chinese). Chin. J. Integr. Trad. West Med. 38, 272-284. doi: 10.7661/j.cjim.20180119.038

Chen, W., Zhong, S., Wang, J., Dong, J., Wang, X., Wang, J., et al. (2008). Effect of heart-protecting musk pill on expression of bcl-2, caspase- 3 in cerebral ischemia reperfusion injury in rats. J. Emerg. Tradit. Chin. Med. 17, 1421-1446.

Chen, Z., and Ma, L. (2013). Effects of Shexiangbaoxin pill on inflammation indicators, cardiac and vascular endothelial function in patients with acute myocardial infarction. J. Chongqing Med. Univ. 38, 912-914. doi: 10.11699/ cyxb20130823

Ding, R., Gui, Y., Chen, J., and Wu, Z. (2013). Effect of Shexiangbaoxin pills on heart function in patients with chronic ischemia heart failure. Acad. J. Second Military Med. Univ. 34, 37-40. doi: 10.3724/SP.J.1008.2013.00037

Dong, T., Wang, J., Ma, X., Ma, R., Wen, J., Chen, N., et al. (2018). Shexiang Baoxin pills as an adjuvant treatment for chronic heart failure: a system review and meta-analysis. Evid. Based Complement. Altern. Med. 2018:6949348. doi: 10.1155/2018/6949348

Dong, Y., Liu, C., Zhao, Y., Ponnusamy, M., Li, P., and Wang, K. (2018). Role of noncoding RNAs in regulation of cardiac cell death and cardiovascular diseases. Cell Mol. Life. Sci. 75, 291-300. doi: 10.1007/s00018-017-2640-8 low methodologic quality, ill-defined bias risk, and short-term follow-ups, which to some extent decreases the credibility of the clinical data. Therefore, randomized, controlled, and doubleblind trials with large sample sizes and long-term follow-ups are urgently needed to affirm efficacy and prospect of SBP in CVDs management.

\section{AUTHOR CONTRIBUTIONS}

LL and XG designed this study and wrote the manuscript. XS prepared the figures and tables. CC and YQ compiled relevant research and studies.

\section{FUNDING}

This study was supported by the grants from the National Natural Science Foundation of China (No. 81270353).

Durgin, B. G., and Straub, A. C. (2018). Redox control of vascular smooth muscle cell function and plasticity. Lab. Invest. 98, 1254-1262. doi: 10.1038/s41374018-0032-9

Fan, W., Wu, Z., and Shi, H. (2018). Expert consensus of treating coronary heart disease angina by Shenxiang Baoxin Pill (Chinese). Chin. J. Integr. Trad. West Med. 38, 145-153. doi: 10.7661/j.cjim.20170801.307

Fan, X., Shi, M., Wang, Y., Liang, Q., and Luo, G. (2011). Transcriptional profiling analysis of HMP-treated rats with experimentally induced myocardial infarction. J. Ethnopharmacol. 137, 199-204. doi: 10.1016/j.jep.2011.05.010

Fang, H. Y., Zeng, H. W., Lin, L. M., Chen, X., Shen, X. N., Fu, P., et al. (2017). A network-based method for mechanistic investigation of Shexiang Baoxin Pill's treatment of cardiovascular diseases. Sci. Rep. 7:43632. doi: 10.1038/srep43632

Feng, Z., Lv, X., Zhao, Z., and Gu, H. (2017). Impact of Wenxin granule combined with Shexiang-Baoxin pills on ectopic beats and heart rate variability in arrhythmia patients caused by AMI. Pract. J. Cardiac. Cereb. Pneum. Vasc. Dis. $25,100-103$.

Forouzanfar, M. H., Liu, P., Roth, G. A., Ng, M., Biryukov, S., Marczak, L., et al. (2017). Global burden of hypertension and systolic blood pressure of at least 110 to $115 \mathrm{~mm} \mathrm{Hg}, 1990-2015$. JAMA 317, 165-182. doi: 10.1001/jama.2016.19043

Gimbrone, M. A. Jr., and Garcia-Cardena, G. (2016). Endothelial cell dysfunction and the pathobiology of atherosclerosis. Circ. Res. 118, 620-636. doi: 10.1161/ CIRCRESAHA.115.306301

Guo, D., Murdoch, C. E., Liu, T., Qu, J., Jiao, S., Wang, Y., et al. (2018). Therapeutic angiogenesis of Chinese herbal medicines in ischemic heart disease: a review. Front. Pharmacol. 9:428. doi: 10.3389/fphar.2018.00428

Hao, P., Jiang, F., Cheng, J., Ma, L., Zhang, Y., and Zhao, Y. (2017). Traditional Chinese medicine for cardiovascular disease: evidence and potential mechanisms. J. Am. Coll. Cardiol. 69, 2952-2966. doi: 10.1016/j.jacc.2017.04. 041

Hu, S., Tian, D., Chen, G., Liu, J., Ke, Y., and Bian, K. (2009). Protective effect of heart-protecting musk pill on heart in spontaneously hypertensive rats. Lishizhen Med. Mater. Med. Res. 20, 2458-2461. doi: 10.2147/IJGM.S15235

Hu, Y. F., Chen, Y. J., Lin, Y. J., and Chen, S. A. (2015). Inflammation and the pathogenesis of atrial fibrillation. Nat. Rev. Cardiol. 12, 230-243. doi: 10.1038/ nrcardio.2015.2

Huang, F., Liu, Y., Yang, X., Che, D., Qiu, K., Hammock, B. D., et al. (2017). Shexiang Baoxin pills promotes angiogenesis in myocardial infarction rats via up-regulation of 20-HETE-mediated endothelial progenitor cells mobilization. Atherosclerosis 263, 184-191. doi: 10.1016/j.atherosclerosis.2017.06.012

Huang, M., Wu, J., and Huang, G. (2017). Impact of Shexiang-baoxin pills on exercise tolerance and cardiopulmonary function of heart failure patients with normal ejection fraction. Pract. J. Cardiac. Cereb. Pneum. Vasc. Dis. 25, 87-90. 
Huang, H., Yang, Y., Lv, C., Chang, W., Peng, C., Wang, S., et al. (2015). Pharmacokinetics and tissue distribution of five bufadienolides from the Shexiang Baoxin Pill following oral administration to mice. J. Ethnopharmacol. 161, 175-185. doi: 10.1016/j.jep.2014.07.056

Huang, J., Hong, X., Xu, W., Chen, J., and Zhang, S. (2011). Effect of heartprotecting musk pill on myocardial interstitium reconstruction and the expression of MMP-9/TIMP-1 mRNA in rabbits with heart failure after myocardial infarction. Chin. Tradit. Pat. Med. 33, 1116-1121.

Huang, L. (2009). Effect of Shexiangbaoxin Pills on old age of patients with isolated systolic hypertension. Int. Med. Health Guid. News 15, 69-71. doi: 10.3760/cma. j.issn.1007-1245.2009.10.027

Huang, Y. (2017). Effects of Shexiang Baoxin Pills combined with trimetazidine on vascular endothelial function and plasma BNP in elderly patients with coronary heart disease and heart failure. J. Hubei. Univ. Chin. Med. 19, 22-25. doi: 10.3969/j.issn.1008-987x.2017.01.06

Hurtubise, J., McLellan, K., Durr, K., Onasanya, O., Nwabuko, D., and Ndisang, J. F. (2016). The different facets of dyslipidemia and hypertension in atherosclerosis. Curr. Atheroscler. Rep. 18:82. doi: 10.1007/s11883-0160632-z

Jiang, B., Cai, F., Gao, S., Meng, L., Liang, F., Dai, X., et al. (2012). Induction of cytochrome P450 3A by Shexiang Baoxin Pill and its main components. Chem. Biol. Interact. 195, 105-113. doi: 10.1016/j.cbi.2011.12.001

Jiang, F., Lin, X., Jia, J., Xu, X., and Liu, B. (2017). Meta-analysis of trimexazine combined with Shexiang Baoxin pills in the treatment of unstable angina. Chin. J. Evid. Based Cardiovasc. Med. 9, 528-538. doi: 10.3969/j.issn.1674-4055.2017. 05.05

Jiang, P., Dai, W., Yan, S., Chen, Z., Xu, R., Ding, J., et al. (2011). Biomarkers in the early period of acute myocardial infarction in rat serum and protective effects of Shexiang Baoxin Pill using a metabolomic method. J. Ethnopharmacol. 138, 530-536. doi: 10.1016/j.jep.2011.09.049

Jiang, P., Liu, R., Dou, S., Liu, L., Zhang, W., Chen, Z., et al. (2009). Analysis of the constituents in rat plasma after oral administration of Shexiang Baoxin pill by HPLC-ESI-MS/MS. Biomed. Chromatogr. 23, 1333-1343. doi: 10.1002/bmc. 1258

Kong, F., Wei, Z., Fu, R., Liu, J., Huang, J., Deng, J., et al. (2015). Effective observation on treating myocardial infarction plus left ventricular failure by EECP plus th Shexiang Baoxin Wan. Clin. J. Chin. Med. 7, 15-20. doi: 10.3969/ j.issn.1674-7860.2015.13.007

Li, G., Chen, Y., and Wu, J. (2015). Promotion of function of endothelial progenitor cells with Shenxiang Baoxin pill Treatment under shear stress. J. Biomed. Eng. 32, 847-853. doi: 10.7507/1001-5515.20150152

Li, R., Wu, T., Zhu, C., Huang, G., and Peng, L. (2015). Clinical Observation on Shexiang Baoxin pill in the treatment of chronic heart failure induced by coronary heart disease. Chin. J. Integr. Med. Cardio. 13, 1704-1706. doi: 10.3969/j.issn.1672-1349.2015.15.003

Li, Y., Guo, H., Ma, H., and Luo, H. (2015). Effect of Shexiang Baoxin Pills on pioglitazone on the expression of NF- $\kappa$ B, PPAR- $\alpha$ and PPAR- $\gamma$ in rats with metabolic syndrome. J. Liaoning Univ. Tradit. Chin. Med. 17, 57-59. doi: 10. 13194/j.issn.1673-842x.2015.05.019

Li, H., Li, J., Wu, B., Xie, K., Gao, W., Shen, Y., et al. (2018). Effect of Shexiang Baoxin Pill on coronary blood flow after selective percutaneous coronary intervention: a double-blinded, randomized, controlled clinical trial. Chin. J. Integr. Trad. West Med. 38, 295-299.

Li, T., Li, Y., and Fan, W. (2006). Effects of She Xiang Bao Xin Pill and simvastatin on stability of atherosclerotic plaque in rabbit femoral artery. Chin. J. Geriatr. Heart Brain Ves. Dis. 8, 296-299.

Li, Y., Cao, F., Liu, F., Bai, X., and Lu, Y. (2012). Effects of Shexiangbaoxin pills on the expression of cardiac $\alpha 1$ - and $\beta$-adrenergic receptor subtypes in rat hearts with heart failure induced by myocardial infarction. Chin. Med. J. 125, 1556-1562. doi: 10.3760/cma.j.issn.0366-6999.2012.09.007

Li, Y., Luo, X., Fan, W., and Dai, R. (1999). Effect of heart-protecting musk pill on nitric oxide metabolism of arterial wall in cholesteriol-fed rabbits. Chin. J. Crit. Care Med. 19, 451-453.

Liu, C., and Huang, Y. (2016). Chinese herbal medicine on cardiovascular diseases and the mechanisms of action. Front. Pharmacol. 7:469. doi: 10.3389/fphar. 2016.00469

Liu, J., Li, J., Wang, C., Li, Y., Zhu, Z., and Xu, X. (2017). Analysis of the effect of losartan plus Shexiang Baoxin pill on patients of hypertnesion combined with heart failure. Progr. Mod. Biomed. 17, 3317-3319. doi: 10.13241/j.cnki. pmb.2017.17.029

Liu, R., Runyon, R. S., Wang, Y., Oliver, S. G., Fan, T. P., and Zhang, W. (2015). Deciphering ancient combinatorial formulas-the Shexiang Baoxin pill. Science 347(Suppl. 6219), S40-S42.

Liu, S., Yang, Y., Jiang, S., Tang, N., Tian, J., Ponnusamy, M., et al. (2018). Understanding the role of non-coding RNA (ncRNA) in stent restenosis. Atherosclerosis 272, 153-161. doi: 10.1016/j.atherosclerosis.2018. 03.036

Liu, X., Wang, Y., and Han, Y. (2012). Effect of Shexiangbaoxinwan Pill on myocardial fibrosis of diabetic rats. Pharmacol. Clin. Chin. Mater. Med. 28, 28-31. doi: 10.13412/j.cnki.zyyl.2012.01.013

Liu, Y. (2016). Observation of efficacy and safety of heart-protecting musk pills in the treatment of elderly patients with acute ST segment elevation myocardial infarction after percutaneous coronary intervention. Med. J. Chin. Peop. Health 28, 7-9. doi: 10.3969/j.issn.1672-0369.2016.21.003

Luo, Y. (2015). Influence of Shexiangbaoxin pills on QT interval dispersion in acute myocardial infarction patients. Chin. J. Mod. Drug Appl. 9, 37-39. doi: 10.14164/j.cnki.cn11-5581/r.2015.15.020

Lv, C., Chen, L., Fu, P., Yang, N., Liu, Q., Xu, Y., et al. (2017). Simultaneous quantification of 11 active constituents in Shexiang Baoxin Pill by ultraperformance convergence chromatography combined with tandem mass spectrometry. J. Chromatogr. B Analyt. Technol. Biomed. Life Sci. 1052, 135-141. doi: 10.1016/j.jchromb.2017.03.033

Lv, C., Huang, H., Chang, W., and Liu, R. (2014). Screeing of active compounds of pro-angiogenic in Shexiang Baoxin pill. J. Pharm. Pract. 32, 344-351. doi: 10.3969/j.issn.1006-0111.2014.05.007

Ma, L., Wu, Y., Wang, W., and Chen, W. (2018). Interpretation of the report on cardiovascular diseases in China (2017). Chin. J. Cardiovasc. Med. 23, 3-6. doi: 10.3969/j.issn.1007-5410.2018.01.002

Moslehi, J., Amgalan, D., and Kitsis, R. N. (2017). Grounding cardiooncology in basic and clinical science. Circulation 136, 3-5. doi: 10.1161/ CIRCULATIONAHA.117.025393

Ning, R., Xu, C., Chai, D., and Lin, J. (2011). Effect of Shengxiang Baoxin Pills on cell proliferation and oxidative stress induced by $\mathrm{H} 2 \mathrm{O} 2$ in human umbilicial vein endothelial cells. Chin. J. Integr. Med. Cardio. 9, 966-969.

Oparil, S., Acelajado, M. C., Bakris, G. L., Berlowitz, D. R., Cifkova, R., Dominiczak, A. F., et al. (2018). Hypertension. Nat. Rev. Dis. Primers 4:18014. doi: 10.1038/ nrdp. 2018.14

Peng, C., Yang, Y., Lv, C., Jin, H., Tao, J., Yuan, X., et al. (2015). Pharmacokinetic study of five ginsenosides using a sensitive and rapid liquid chromatographytandem mass spectrometry method following single and multiple oral administration of Shexiang Baoxin pills to rats. Biomed. Chromatogr. 29, 425436. doi: 10.1002/bmc.3293

Qi, C., Zhou, Z., Wang, Y., Zhang, X., and Xiang, D. (2017). Clinical study on Shexiang Baoxin Pill in the treatment of doxorubicin-induced cardiotoxicity. Mod. Oncol. 25, 3323-3326. doi: 10.3969/j.issn.1672-4992.2017.20.031

Roth, G. A., Johnson, C., Abajobir, A., Abd-Allah, F., Abera, S. F., Abyu, G., et al. (2017). Global, regional, and national burden of cardiovascular diseases for 10 Causes, 1990 to 2015. J. Am. Coll. Cardiol. 70, 1-25. doi: 10.1016/j.jacc.2017. 04.052

Shapiro, M. D., and Fazio, S. (2016). From lipids to inflammation: new approaches to reducing atherosclerotic risk. Circ. Res. 118, 732-749. doi: 10.1161/ CIRCRESAHA.115.306471

Shen, W., Fan, W., Shi, H., Li, Y., Luo, X., Zhang, X., et al. (2010). Effects of Shexiang Baoxin Pill on angiogenesis in atherosclerosis plaque and ischemic myocardium. Chin. J. Integr. Trad. West Med. 30, 1284-1287.

Shen, Z., Wang, Y., Guo, W., Yao, Y., and Wang, X. (2016). Potential herbdrug interaction of shexiang baoxin pill in vitro based on drug metabolism transporter. Am. J. Transl. Res. 8, 5545-5556.

Siti, H. N., Kamisah, Y., and Kamsiah, J. (2015). The role of oxidative stress, antioxidants and vascular inflammation in cardiovascular disease (a review). Vascul. Pharmacol. 71, 40-56. doi: 10.1016/j.vph.2015.03.005

Sun, L. L., Li, W. D., Lei, F. R., and Li, X. Q. (2018). The regulatory role of microRNAs in angiogenesis-related diseases. J. Cell Mol. Med. 22, 4568-4587. doi: $10.1111 / \mathrm{jcmm} .13700$

Tao, J., Jiang, P., Peng, C., Li, M., Liu, R., and Zhang, W. (2016). The pharmacokinetic characters of simvastatin after co-administration with 
Shexiang Baoxin Pill in healthy volunteers' plasma. J. Chromatogr. B Analyt. Technol. Biomed. Life Sci. 1026, 162-167. doi: 10.1016/j.jchromb.2016.01.018

Tao, J., Mao, L., Zhou, B., Liu, Q., Yang, A., Wei, G., et al. (2017). Simultaneous determination of ginsenosides and bufadienolides in rat plasma after the oral administration of Shexiang Baoxin Pill for pharmacokinetic study by liquid chromatography tandem mass spectrometry following solid-phase extraction. Biomed. Chromatogr. 31:e3816. doi: 10.1002/bmc.3816

Tao, Y., Li, J., Wei, Y., and Huang, Q. (2015). Experimental study of Shexiang Baoxin Pill in preventing and treating atherosclerosis. Tradit. Chin. Drug Res. Pharmacol. 26, 508-511. doi: 10.3969/j.issn.1003-9783.2015.04.020

Tian, D., Ling, S., Chen, G., Li, Y., Liu, J., Ferid, M., et al. (2011). Hypertensive nephropathy treatment by heart-protecting musk pill: a study of antiinflammatory therapy for target organ damage of hypertension. Int. J. Gen. Med. 4, 131-139. doi: 10.2147/IJGM.S15235

Ungvari, Z., Tarantini, S., Kiss, T., Wren, J. D., Giles, C. B., Griffin, C. T., et al. (2018). Endothelial dysfunction and angiogenesis impairment in the ageing vasculature. Nat. Rev. Cardiol. 15, 555-565. doi: 10.1038/s41569-018-0030-z

Wang, S., Li, Y., Fan, W., and Dai, R. (2002). The stimulate angiogenesis effect of Shenxiangbaoxin Pills on the heart of myocardial infarction rats. Chin. Tradit. Pat. Med. 24, 446-449.

Wei, D., Zheng, N., Zheng, L., Wang, L., Song, L., and Sun, L. (2018). Shexiang Baoxin pill corrects metabolic disorders in a rat model of metabolic syndrome by targeting mitochondria. Front. Pharmacol. 9:137. doi: 10.3389/fphar.2018. 00137

Wei, S., Zhan, C., and Deng, Z. (2015). Overview of safety studies of Shexiang Baoxin Pill (Chinese). Chin. Tradit. Pat. Med. 37, 1080-1082. doi: 10.3969/j. issn.1001-1528.2015.05.034

Wu, B., Li, J., Jin, B., Li, H., Shi, H., and Luo, X. (2018). Pro-angiogenic mechanisms of Shexiang Baoxin pills (Chinese). Chin. Tradit. Pat. Med. 40, 1384-1388. doi: 10.3969/j.issn.1001-1528.2018.06.034

Xiang, L., Jiang, P., Wang, S., Hu, Y., Liu, X., Yue, R., et al. (2013). Metabolomic strategy for studying the intervention and the synergistic effects of the shexiang baoxin pill for treating myocardial infarction in rats. Evid. Based Complement. Altern. Med. 2013:823121. doi: 10.1155/2013/823121

Xiang, L., Jiang, P., Zhan, C., Chen, Z., Liu, X., Huang, X., et al. (2012). The serum metabolomic study of intervention effects of the traditional Chinese medicine Shexiang Baoxin Pill and a multi-component medicine polypill in the treatment of myocardial infarction in rats. Mol. Biosyst. 8, 2434-2442. doi: $10.1039 / \mathrm{c} 2 \mathrm{mb} 25172 \mathrm{~h}$

Xie, Z., and Wang, C. (2016). Effects of Shexiang Baoxin Pills on serum hs-CRP and MMP-9 in patients with unstable angina pectoris. Chin. J. Integr. Med. Cardio. 14, 804-806. doi: 10.3969/j.issn.1672-1349.2016.08.002

Xing, J., Li, L., Li, R., and Wang, H. (2015). Clinical efficacy observation of Shexiang Baoxin pills on unstable angina. Tianjin J. Tradit. Chin. Med. 32, 530-532. doi: 10.11656/j.issn.1672-1519.2015.09.05

$\mathrm{Xu}, \mathrm{B}$., Zeng, K., and Huang, Y. (2015). Effect of Shexiang Baoxin Pill on time difference expression of IL-18 in border zone myocardium and ventricular arrhythmia of rats with acute myocardial infarction. Chin. Tradit. Herbal Drugs 46, 1195-1199. doi: 10.7501/j.issn.0253-2670.2015.08.018

Xu, C., Ning, R., Chai, D., and Lin, J. (2011). Effect of Shexiang Baoxin Wan on cell apoptosis and expression of inflammatory factors in human umbilicial vein endothelial cells induced with hydrogen peroxide. Chin. J. Arterioscler. 19, 813-818.

Xu, M., and Zhang, Z. (2016). The effects and mechanisms of Shexiangbaoxinwan on myocardial fibrosis in rats with pressure overload. Pharmacol. Clin. Chin. Mater. Med. 32, 128-132. doi: 10.13412/j.cnki.zyyl.2016.03.034

Yang, B., Zhou, C., Hu, Y., Zhang, D., and Xiao, J. (2015). Effects and related mechanisms study on heart protecting musk pill to the inhibition of inflammatory reaction of ventricular remodeling with acute myocardial infarction model in rats. Chin. J. Basic Med. Tradit. Chin. Med. 21, 285-290.
Yang, F., Jiang, X., and Shen, Y. (2015). Clinical study on Shexiang Baoxin Pills combined with atorvastatin in treatment of acute myocardial infarction. Drug Clin. 30, 1087-1090. doi: 10.7501/j.issn.1674-5515.2015.09.009

Yang, H., and Zhang, Y. (2016). Influence of Shexiang Baoxin Pills on hemorhelogy and endothelial function in patients with unstable angina. Chin. Pharm. 25, $70-72$.

Yang, Y., Chen, W., Sun, X., Wang, X., Li, Z., Liang, M., et al. (2016). Shexiang baoxin pill protects cardiomyocytes against high glucose-induced injury by inhibiting the p38 MAPK and NF-кB pathway. Chin. J. Clin. Anat. 34, 517-527. doi: 10.13418/j.issn.1001-165x.2016.05.009

Yu, J., Gao, C., and Ren, Y. (2012a). Effect of Shexiang Baoxin pill on abdominal aortic atherosclerosis in rabbits. J. Chin. Pract. Diagn. Treat. 26, 852-856.

Yu, J., Gao, C., Xu, W., and Zhang, L. (2012b). To investigate the effect of Shexiang Baoxin Wan (SXBXW) preventing in-stent restenosis after stenting. China Pract. Med. 7:23. doi: 10.14163/j.cnki.11-5547/r.2012.23.027

Yuan, X., Han, L., Fu, P., Zeng, H., Lv, C., Chang, W., et al. (2018). Cinnamaldehyde accelerates wound healing by promoting angiogenesis via up-regulation of PI3K and MAPK signaling pathways. Lab. Invest. 98, 783-798. doi: 10.1038/s41374018-0025-8

Zhang, K. J., Zhu, J. Z., Bao, X. Y., Zheng, Q., Zheng, G. Q., and Wang, Y. (2017). Shexiang Baoxin Pills for coronary heart disease in animal models: preclinical evidence and promoting angiogenesis mechanism. Front. Pharmacol. 8:404. doi: 10.3389/fphar.2017.00404

Zhang, L., Liu, N., Zhang, J., and Zhang, H. (2016). Effect of Shexiang baoxin pills on clopidogrel resistance in patients with acute coronary syndrome. Pak. J. Pharm. Sci. 29(6 Suppl.), 2303-2306.

Zhang, Y., Lu, Y., Zhu, X., and Jiang, Y. (2016). Effect of Shexiang Baoxin pill on oxidative stress and endothelial dysfunction in patients with hypertension with left ventricular hypertrophy. Mod. J. Integr. Tradit. Chin. West Med. 25, 1718-1725. doi: 10.3969/j.issn.1008-8849.2016.16.003

Zhang, Q., Gao, R., Chong, B. H., Liang, S. X., Jiang, X., and Che, X. (2014). Influence of Shexiang Baoxin Pill on vascular smooth muscle cell proliferation and phenotypic transformation. Chin. Arch. Tradit. Chin. Med. 32, 906-910. doi: 10.13193/j.issn.1673-7717.2014.04.068

Zhang, X., and Guan, A. (2016). Effect of Shexiang Baoxin pill on serum indicators of myocardial injury and heart function in patients with acute ST segment elevation myocardial infarction after PCI. Med. J. Integr. Tradit. Chin. West Med. 25, 3772-3775. doi: 10.3969/j.issn.1008-8849.2016.34.004

Zhang, Y. (2013). Influence of Shexiang Baoxin Pills on oxidative stress in patients with unstable angina pectoris of coronary heart disease. Chin. J. Exp. Tradit. Med. Formul. 19, 334-336. doi: 10.11653/syfj2013100334

Zhou, Z., Shen, W., Yu, L., Xu, C., and Wu, Q. (2016). A Chinese patent medicine, Shexiang Baoxin Pill, for Non-ST-elevation acute coronary syndromes: a systematic review. J. Ethnopharmacol. 194, 1130-1139. doi: 10.1016/j.jep.2016. 11.024

Zhu, L., Dong, Q., Liu, Z., Yang, Y., Zhou, S., and Dong, B. (2008). Effects of Shexiang Baoxin Pill on LOX-1 gene and protein expression in rabbits of atherosclerosis. Acta Univ. Tradit. Med. Sin. Pharmacol. Shanghai 22, 43-45.

Conflict of Interest Statement: The authors declare that the research was conducted in the absence of any commercial or financial relationships that could be construed as a potential conflict of interest.

Copyright (c) $2018 \mathrm{Lu}$, Sun, Chen, Qin and Guo. This is an open-access article distributed under the terms of the Creative Commons Attribution License (CC BY). The use, distribution or reproduction in other forums is permitted, provided the original author(s) and the copyright owner(s) are credited and that the original publication in this journal is cited, in accordance with accepted academic practice. No use, distribution or reproduction is permitted which does not comply with these terms. 